\title{
Covariance imaging experiments using a pixel-imaging mass-spectrometry camera
}

\author{
Craig S. Slater, Sophie Blake, Mark Brouard, and Alexandra Lauer \\ Department of Chemistry, University of Oxford, The Physical and Theoretical Chemistry Laboratory, South Parks Road, \\ Oxford OX1 3QZ, United Kingdom
}

\author{
Claire Vallance \\ Department of Chemistry, University of Oxford, Chemistry Research Laboratory, Mansfield Road, Oxford OXI 3TA, United Kingdom \\ Jaya John John \\ Department of Physics, University of Oxford, Denys Wilkinson Building, Keble Road, Oxford OXI 3RH, United Kingdom
}

Renato Turchetta

Rutherford Appleton Laboratory, Chilton, Didcot OX11 OQX, United Kingdom

Andrei Nomerotski

Department of Physics, Brookhaven National Laboratory, Upton, New York 11973, USA

Lauge Christensen and Jens H. Nielsen ${ }^{\dagger}$

Department of Physics and Astronomy, Aarhus University, DK-8000 Aarhus C, Denmark

Mikael P. Johansson

Department of Chemistry, Laboratory for Instruction in Swedish, University of Helsinki, A.I. Virtanens plats 1, P.O. Box 55, FI-00014 Helsinki, Finland

\author{
Henrik Stapelfeldt ${ }^{\ddagger}$ \\ Department of Chemistry and Interdisciplinary Nanoscience Center (iNANO), Aarhus University, DK-8000 Aarhus C, Denmark \\ (Received 9 October 2013; published 21 January 2014)
}

\begin{abstract}
The "pixel imaging mass spectrometry" camera is used to perform femtosecond laser-induced Coulomb explosion imaging of 3,5-dibromo-3', $5^{\prime}$-difluoro- $4^{\prime}$-cyanobiphenyl molecules prealigned in space. The experiment allows the concurrent detection of the correlated two-dimensional momentum images of all the ionic fragments resulting from fragmentation of multiple molecules in each acquisition cycle. The Coulomb explosion studies provide rich information about the parent molecular structure and fragmentation dynamics, and open new opportunities for real-time imaging of intramolecular processes.
\end{abstract}

DOI: 10.1103/PhysRevA.89.011401

PACS number(s): 33.80.Gj, 34.50.Gb

Coulomb explosion imaging (CEI) is a method aimed at determining molecular structure. Several valence electrons in a molecule are rapidly removed and the multiply charged cation thereby generated subsequently fragments due to internal electrostatic repulsion. The resulting ions are then detected, and their final momenta recorded. Although a variety of methods have been used to induce Coulomb explosion [1-4], the most widespread method relies on irradiation of neutral molecules by tabletop intense ultrashort laser pulses, typically in the visible or near infrared region [5-7]. This renders Coulomb explosion possible for a broad range of molecules and, importantly, it enables timing of the event such that studies of time-resolved dynamics become possible through pump-probe measurements [8-10].

\footnotetext{
*mark.brouard@chem.ox.ac.uk

${ }^{\dagger}$ Present address: Research Software Development Team, Research Computing and Facilitating Services, University College London, Gower Street, London, WC1E 6BT, United Kingdom.

${ }^{\ddagger}$ henriks@chem.au.dk
}

Using CEI to extract structure of molecules requires the measurement of the momenta of the ionic fragments and their correlations. The predominant method has been the detection of the full three-dimensional (3D) momentum vectors of all fragments from each individual breakup process, i.e., from each molecule that undergoes Coulomb explosion. This is possible through the use of multiparticle 3D imaging detectors and coincidence analysis [11-14]. The information content accessible in such measurements is extremely high and has recently enabled the determination of the absolute configuration of small chiral molecules [7]. However, in practice, various factors limit the use of 3D momentum imaging coincidence detectors to CEI studies of rather small molecules, typically containing fewer than ten atoms.

Our goal here is to introduce an alternative approach to laser-induced CEI aimed particularly at molecules larger than the ones studied so far. It combines the multihit capability of the "pixel imaging mass spectrometry" (PImMS) camera $[15,16]$ to record $m / z$-specific two-dimensional momentum images of all fragment ions with the ability to place molecules in a well-defined spatial alignment $[10,17]$ prior 
to the Coulomb explosion process. Since the ion signal per laser shot typically contains contributions from more than one molecule, the correlations between the momenta of the fragment ions is obtained through statistical analysis. The method is illustrated through CEI experiments on prealigned 3,5dibromo-3', 5' -difluoro-4'-cyanobiphenyl (BFCbP) molecules. The current work builds on a previous study of a similar molecule by Stapelfeldt and co-workers [10]. Here we show that the use of the PImMS camera, instead of the conventional CCD camera employed previously, has wide-ranging consequences in allowing the correlation of momenta of fragments of different masses to be recorded. These data provide rich information about the structure of the parent molecule and on the fragmentation dynamics. Only a small fraction of these correlated data is presented in this communication, the bulk of which will be published elsewhere. In that work we will also present a detailed comparison of the experimental results with simulations of the Coulomb explosion dynamics.

Apart from the PImMS camera, the experimental setup has been described in Ref. [10]. A solid sample of BFCbP was heated to $170^{\circ}$ in $\mathrm{He}$ and the mixture expanded into vacuum through a pulsed valve to form a cold molecular beam. An electrostatic deflector was employed to select molecules in low rotational quantum states to aid alignment of the molecules. The BFCbP molecule, illustrated in Fig. 1, consists of two substituted phenyl rings joined by a $\mathrm{C}-\mathrm{C}$ single bond.

To optimize the information content of the fragment ion momentum images, the molecules were one-dimensional (1D) adiabatically aligned using a linearly polarized $10 \mathrm{~ns}$ (FWHM) pulse from a Nd:YAG laser [yttrium aluminum garnet (YAG)

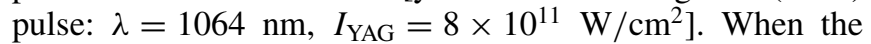
YAG pulse is polarized perpendicular to the detector the most polarizable molecular axis, which coincides with the $C_{2}$ symmetry axis, is confined along this direction. Thus, the molecules are imaged in end view, and the relative velocities of the $\mathrm{Br}^{+}$and $\mathrm{F}^{+}$ions are indicative of the dihedral angle. Alternatively, with the YAG pulse polarized parallel to the detector the molecules are imaged in side view, as shown in Fig. 1. In this case the velocity of the $\mathrm{N}^{+}$ion is well suited as a reference with which the velocities of the other fragment ions may be compared.

Coulomb explosion was induced by a 30 fs (FWHM) probe pulse $\left(\lambda=800 \mathrm{~nm}, I_{\text {probe }}=3 \times 10^{14} \mathrm{~W} / \mathrm{cm}^{2}\right)$, which

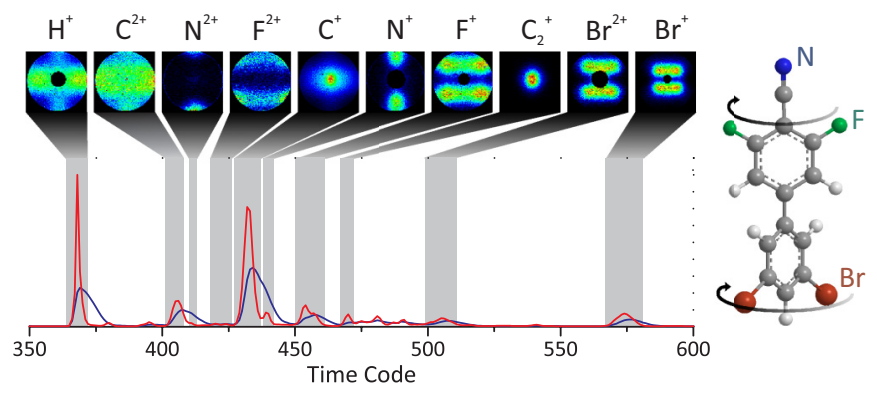

FIG. 1. (Color online) Time-of-flight mass spectrum, in time codes each of $12.5 \mathrm{~ns}$ duration, for the BFCbP molecule in side-view alignment (see text), before (blue) and after (red) postprocessing. The conventional ion images for each assigned mass peak are shown at the top of the figure. The BFCbP molecule is illustrated on the right. was overlapped in time and space with the YAG pulse. Its polarization was linear and kept in the same direction as the YAG pulse. The fragment ions produced by the probe pulse were accelerated by a three-electrode ion optics assembly, optimized to effect velocity-map [18] ion imaging (VMI) [19], and focused onto a detector consisting of a pair of microchannel plates (MCPs) and a P47 phosphor screen. The ion images were recorded at $20 \mathrm{~Hz}$, limited by the repetition rate of the YAG laser.

Events on the phosphor screen were imaged by the PImMS camera [20-22], a pixelated event-counting sensor tailored for applications in time-of-flight (TOF) imaging mass spectrometry [15,20-22]. As a photon detector, the PImMS camera can replace the $\mathrm{CCD}$ camera commonly used in conventional ion or electron imaging experiments [23,24]. The PImMS1 sensor employed here consists of an array of $72 \times 72$ pixels, each receiving a global time code signal incrementing at a clock cycle of $12.5 \mathrm{~ns}$, which may be synchronized with an external trigger. When a sufficient number of photons impact on a pixel, such that the signal exceeds a set threshold, the current value of the time code is stored. Each pixel has four memory registers, and therefore it is possible to record up to four ion events per pixel per acquisition cycle. This is a sufficient number to detect all ions under typical operating conditions in the present experiments. The data are read out at the end of each acquisition cycle, giving the $x$ and $y$ coordinates and the time code of each ion event, along with the acquisition cycle number. These design features enable the PImMS camera to be used to measure the velocity vector correlations between numerous photofragments at once in a high count-rate regime.

Every flash on the phosphor screen typically results in the illumination of a cluster of pixels corresponding to each ion detected [see Fig. 2(a)]. The intensity profile within each cluster is expected to have an approximately Gaussian profile, with a higher brightness towards the center of the cluster. For an event to be recorded, the charge buildup within an individual pixel must be sufficient to exceed the chosen threshold value. Pixels at the center of a cluster are subject to a higher photon flux, and therefore tend to go over threshold at an earlier time. Since the earliest a pixel can detect an ion event is the actual time of the ion hit on the detector, the earliest time codes present within a cluster provide the most accurate arrival time information.

An algorithm was therefore employed to search for clusters of spatially connected pixels, and the clusters were reduced to the pixel with the earliest time code. The maximum possible time spread between the earliest and latest time

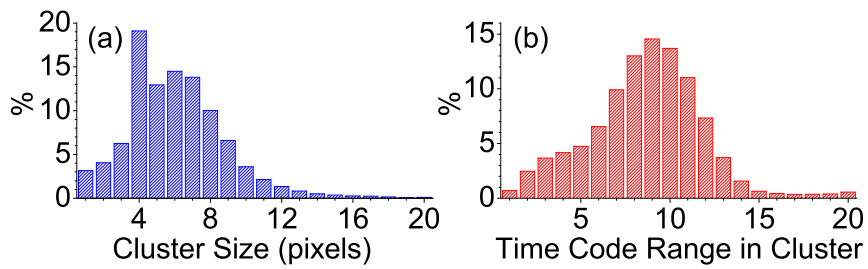

FIG. 2. (Color online) Panel (a) shows the frequency of different cluster sizes in the PImMS data set. Panel (b) characterizes the range of time codes present within the clusters from the earliest to the latest time code observed within each cluster. 
codes within a single cluster was limited to 20 time codes (250 ns). As Fig. 2(b) demonstrates, the majority of data lies within approximately 15 time codes (consistent with the time constant of the P47 phosphor and PImMS pixel electronics), therefore 20 time codes were deemed sufficiently long to collect every pixel belonging to each cluster, and sufficiently short to exclude shadowing of later peaks by earlier ion hits.

Figure 1 shows TOF spectra of BFCbP subsequent to Coulomb explosion, obtained by integrating the signal from the PImMS camera over all pixels both before (blue trace) and after (red trace) postprocessing. The improvement in the quality of the data is striking: The broad features in the raw data are sharpened to well-resolved TOF peaks, limited only by the $12.5 \mathrm{~ns}$ bin width of the PImMS sensor. Note that the time resolution of $12.5 \mathrm{~ns}$ achieved in this experiment was not limited by the $\sim 100$ ns decay time of the P47 phosphor screen, as might be the case when a conventional P47/CCD camera combination is used. This results in a significant improvement in time (and hence mass) resolution without the need to pulse the MCPs.

Correlations between the velocities of the various fragment ions were identified by a covariance analysis, originally developed to correlate mass peaks in a TOF experiment [25-27]. Here we focus on covariances between the measured velocity vectors of the recoiling ions [10,28-30]. The covariance is defined as [31]

$$
\begin{aligned}
\operatorname{cov}(A, B) & =\langle(A-\langle A\rangle)(B-\langle B\rangle)\rangle \\
& =\langle A B\rangle-\langle A\rangle\langle B\rangle,
\end{aligned}
$$

where $\langle i\rangle$ refers to the mean or expectation value of sampled quantity $i$. The covariance is therefore a simple statistical measure of the linear correlation between two variables.

This analysis can be applied to the signal variations in each pixel for two different ionic photofragments, $A$ and $B$. One ionic species is designated as the "reference" ion, and the covariance between each pixel in the reference image and each pixel in the partner image is calculated. In this way, each pixel in the reference image has an associated "covariance map," showing the correlations of this pixel to each pixel in the partner ion image. The covariance maps for each pixel are then rotated such that the reference pixels all lie along a common vector, and summed together. This results in a "recoil"-frame covariance "image," in which the velocity vectors of the reference ions are confined along a single direction, and the covariances with the partner ions are shown relative to this direction.

As an illustration, column I of Fig. 3 shows "raw" ion images of (a) $\mathrm{F}^{+}$and (b) $\mathrm{Br}^{+}$for end-view alignment of the molecules. Both images show a cylindrically symmetric distribution around the alignment axis, as expected, because the molecule is free to rotate around this axis. It is not possible, however, to determine the relationship between the two fragments from the raw ion images alone; a covariance analysis is needed to correlate the velocity vectors of the $\mathrm{F}^{+}$ ions with respect to $\mathrm{Br}^{+}$, or vice versa.

The covariance images shown in column II of Fig. 3 clearly reveal the relationship between the velocities of the $\mathrm{F}^{+}$and $\mathrm{Br}^{+}$ ions. The covariance images consist of four peaks of intensity, with the relative positions of these peaks being determined (a)
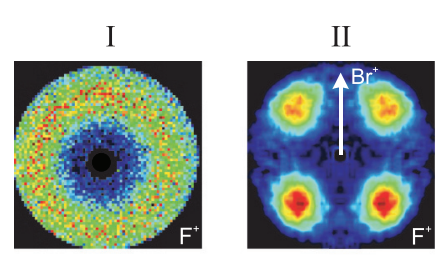

(b)
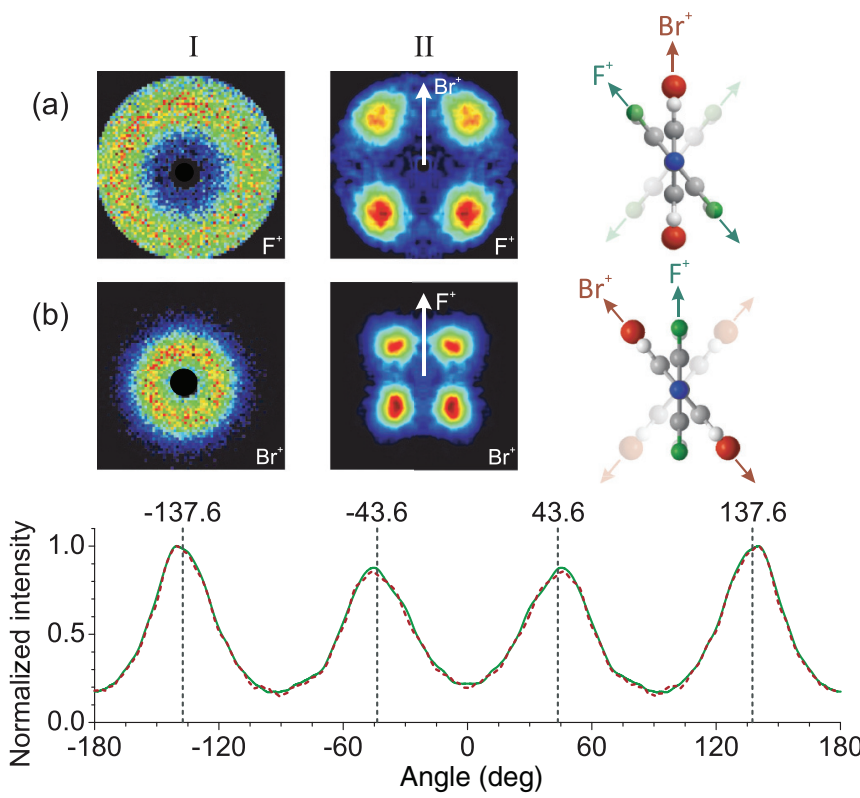

FIG. 3. (Color online) Ion images (I) of (a) $\mathrm{F}^{+}$and (b) $\mathrm{Br}^{+}$, and covariance images (II) of (a) $\mathrm{F}^{+}$relative to $\mathrm{Br}^{+}$, and (b) $\mathrm{Br}^{+}$relative to $\mathrm{F}^{+}$for end-view alignment of the molecules. The molecular reference frames resulting in the covariance maps shown in column II are illustrated on the right. The bottom panel shows the corresponding covariance plotted as a function of the relative angle of $\mathrm{Br}^{+}$to $\mathrm{F}^{+}$ (dashed red), or $\mathrm{F}^{+}$to $\mathrm{Br}^{+}$(continuous green).

by the dihedral angle between the two phenyl rings and the Coulomb explosion dynamics.

The lower panel of Fig. 3 plots the signal intensity from the covariance images as a function of the relative angle between the two ion trajectories. In the limit of axial recoil, the angles of the peaks should be characteristic of the dihedral angle between the two phenyl rings. The equilibrium dihedral angle, computed at density functional theory level following Ref. [32], is $39^{\circ}$. This is in good qualitative agreement with the values extracted from the covariance images, especially considering the rather flat energy surface in the torsional coordinate. The peaks observed for the more distant $\mathrm{Br}^{+}$and $\mathrm{F}^{+}$ion pairs are seen at approximately $\pm 137.6^{\circ}$, which corresponds to a dihedral angle of $42.4 \pm 0.1^{\circ}$ (where the errors represent $2 \sigma$ statistical errors from fits to the data). The peaks on the near side occur at around $\pm 43.6 \pm 0.1^{\circ}$. Notice that the angular distributions derived from covariance images of $\mathrm{Br}^{+}$ relative to $\mathrm{F}^{+}$, and $\mathrm{F}^{+}$relative to $\mathrm{Br}^{+}$should be identical. The very small differences observed (see the lower panel of Fig. 3) reflect limitations in the angular binning of the pixelated covariance data. The recoil angles obtained from the images suggest that the ion trajectories are somewhat affected by Coulomb repulsion between the measured fragment ion pairs, thus slightly increasing the angle observed for the nearest pair. Nevertheless, the results show convincingly that the covariance analysis is a good probe of the dihedral angle, which is a characteristic structural coordinate of the molecule.

Column I of Fig. 4 shows raw ion images of $\mathrm{F}^{+}$and $\mathrm{Br}^{+}$ recorded with side-view alignment of the molecules; images for these and other fragments are also shown in Fig. 1. To some degree, the positions of the substituents within the 
(a)

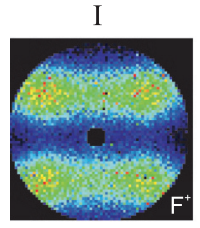

(b)
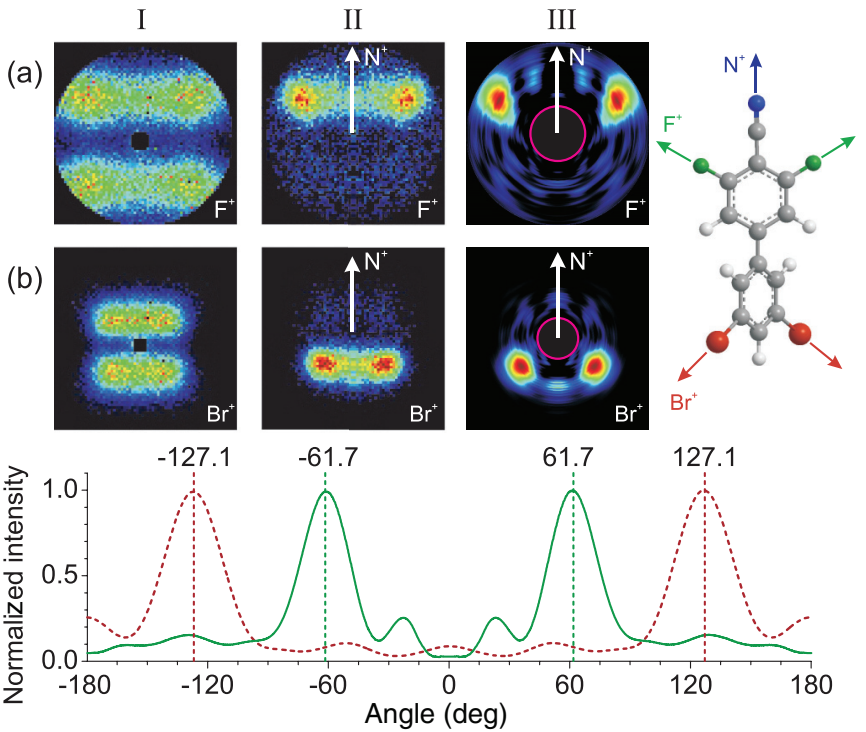

FIG. 4. (Color online) Column I shows (a) $\mathrm{F}^{+}$and (b) $\mathrm{Br}^{+}$images as collected by the PImMS camera with side-view alignment of the molecules. Covariance images of (a) $\mathrm{F}^{+}$and (b) $\mathrm{Br}^{+}$relative to $\mathrm{N}^{+}$ are shown in column II, with the corresponding Abel-inverted images in column III, representing the central slice through a full 3D velocity distribution. The structure shown on the right of the figure illustrates the molecular reference frame resulting in the covariances observed. The resulting angular distributions are plotted for $\mathrm{F}^{+}$relative to $\mathrm{N}^{+}$ (continuous green), and for $\mathrm{Br}^{+}$relative to $\mathrm{N}^{+}$(dashed red).

molecule are reflected in the ion images. Most strikingly, the $\mathrm{N}^{+}$signal (Fig. 1) is directed strongly along the vertical axis, as expected for vertical alignment of the $C_{2}$ symmetry axis. Due to the fourfold symmetry of the images, it is not possible to identify, for instance, whether $\mathrm{Br}$ or $\mathrm{F}$ substituents are located adjacent to the $\mathrm{CN}$ group in the molecule, or on the second phenyl ring. To obtain this information, a covariance analysis is once again required.

Because of the unique position of the $\mathrm{N}$ atom in the molecule, $\mathrm{N}^{+}$has been chosen as the reference ion for the covariance images shown here. Column II of Fig. 4 shows covariance maps of $\mathrm{F}^{+}$and $\mathrm{Br}^{+}$with respect to $\mathrm{N}^{+}$. These covariance images bear a striking resemblance to the molecular structure: $\mathrm{Br}^{+}$is observed on the opposite side of the images relative to $\mathrm{N}^{+}$, while $\mathrm{F}^{+}$is located adjacent to $\mathrm{N}^{+}$, as intuitively expected. The information obtained from the covariance images can be increased by utilizing the cylindrical symmetry that stems from the 1D alignment of the molecules, which allows free rotation around the alignment axis. To retrieve the $3 \mathrm{D}$ velocity distribution we applied the inverse Abel transform, using the pBasex routine [33]. The Abel inverted ion images are displayed in column III of Fig. 4, with the resulting angular distributions of the $\mathrm{Br}^{+}$and $\mathrm{F}^{+}$ ions relative to $\mathrm{N}^{+}$shown in the lower panel. The recoil angles relative to the $\mathrm{N}^{+}$ions are $61.7 \pm 0.2^{\circ}$ for $\mathrm{F}^{+}$, and
$127.1 \pm 0.3^{\circ}$ for $\mathrm{Br}^{+}$. The former angle is close to the bonding angle between the $\mathrm{C}-\mathrm{CN}$ and the $\mathrm{C}-\mathrm{F}$ bonds $\left(60^{\circ}\right)$, whereas the latter angles correspond to $\mathrm{Br}$ ions leaving at an angle of 52.9 with respect to the para $\mathrm{C}-\mathrm{H}$ bond, i.e., somewhat smaller than the $60^{\circ}$ bonding angle between the para $\mathrm{C}-\mathrm{H}$ and the $\mathrm{C}-\mathrm{Br}$ bonds in the parent molecule. Although a more detailed interpretation of the observed angles requires a simulation of the Coulomb explosion process, it is clear that the covariance maps of $\mathrm{F}^{+}$and $\mathrm{Br}^{+}$relative to $\mathrm{N}^{+}$contain valuable structural information about the parent molecule.

The images shown in Figs. 3 and 4 consist of a small subset of the data obtained in these experiments. Covariance maps can be generated for all of the ion fragments assigned in Fig. 1. Thus, a total of around 100 covariance images have been obtained for each parent molecular geometry studied, and most of these show interesting features which can be related qualitatively to the structure of the parent molecule and the subsequent Coulomb explosion dynamics.

Velocity-map imaging employing the PImMS camera, with a covariance analysis of the data, has been demonstrated to be a powerful method for determining correlations between multiple ionic fragments produced following the Coulomb explosion of laser aligned molecules. The current work has been focused on pairwise correlations between selectively chosen fragments. We have demonstrated here that the technique can be used to estimate important structural coordinates within polyatomic molecules, particularly concerning bond angles. Using triple and multicovariance methods [34] should make it possible to extract increasingly detailed molecular structural information. The technique also shows great promise for probing femtosecond time-dependent dynamics induced by a pump pulse prior to the Coulomb explosion pulse. The results of such a time-resolved study will be presented in a future work. The multihit, relatively high count-rate features of PImMS make it ideally suited to the study of the Coulomb explosion dynamics of much larger systems than have hitherto been considered, offering new opportunities for following complex molecular motion, such as that involved in the action of molecular switches or photoisomerization.

The support of the EPSRC via Programme Grant No. EP/G00224X/1 (to M.B. and C.V.), the EU through grant FP7 ITN "ICONIC" (Project Grant No. 238671 to M.B. and C.V.), STFC through a PNPAS award to A.N., M.B., C.V., and R.T., and a mini-IPS grant ST/J002895/1 to A.N. and R.T., ERC through Starting Independent Researcher grant "ImageMS" (C.V.), and a proof of concept grant from ISIS Innovation Ltd. (M.B., C.V., and A.N.) are gratefully acknowledged. A.L. thanks the DFG via Grant No. La 3209/11 for support. The work was supported by the Danish Council for Independent Research (Natural Sciences), The Lundbeck Foundation, and the Carlsberg Foundation. CSC, The Finnish IT Centre for Science, provided computing resources to M.P.J.
[1] Z. Vager, R. Naaman, and E. Kanter, Science 244, 426 (1989).

[2] U. Werner, K. Beckord, J. Becker, and H. O. Lutz, Phys. Rev. Lett. 74, 1962 (1995).
[3] Y. H. Jiang, A. Rudenko, O. Herrwerth, L. Foucar, M. Kurka, K. U. Kühnel, M. Lezius, M. F. Kling, J. van Tilborg, A. Belkacem, K. Ueda, S. Düsterer, R. Treusch, C. D. Schröter, 
R. Moshammer, and J. Ullrich, Phys. Rev. Lett. 105, 263002 (2010).

[4] B. Erk, D. Rolles, L. Foucar, B. Rudek, S. W. Epp, M. Cryle, C. Bostedt, S. Schorb, J. Bozek, A. Rouzee, A. Hundertmark, T. Marchenko, M. Simon, F. Filsinger, L. Christensen, S. De, S. Trippel, J. Küpper, H. Stapelfeldt, S. Wada, K. Ueda, M. Swiggers, M. Messerschmidt, C. D. Schröter, R. Moshammer, I. Schlichting, J. Ullrich, and A. Rudenko, Phys. Rev. Lett. 110, 053003 (2013).

[5] K. Codling and L. Frasinski, J. Phys. B: At., Mol. Opt. Phys. 26, 783 (1993).

[6] J. Gagnon, K. F. Lee, D. M. Rayner, P. B. Corkum, and V. R. Bhardwaj, J. Phys. B: At., Mol. Opt. Phys. 41, 215104 (2008).

[7] M. Pitzer, M. Kunitski, A. S. Johnson, T. Jahnke, H. Sann, F. Sturm, L. P. H. Schmidt, H. Schmidt-Böcking, R. Dörner, J. Stohner, J. Kiedrowski, M. Reggelin, S. Marquardt, A. Schießer, R. Berger, and M. S. Schöffler, Science 341, 1096 (2013).

[8] H. Stapelfeldt, E. Constant, and P. B. Corkum, Phys. Rev. Lett. 74, 3780 (1995).

[9] T. Ergler, A. Rudenko, B. Feuerstein, K. Zrost, C. D. Schröter, R. Moshammer, and J. Ullrich, Phys. Rev. Lett. 97, 193001 (2006).

[10] J. L. Hansen, J. H. Nielsen, C. B. Madsen, A. T. Lindhardt, M. P. Johansson, T. Skrydstrup, L. B. Madsen, and H. Stapelfeldt, J. Chem. Phys. 136, 204310 (2012).

[11] A. Faibis, W. Koenig, E. Kanter, and Z. Vager, Nucl. Instrum. Methods Phys. Res., Sect. B 13, 673 (1986).

[12] J. A. Davies, J. E. LeClaire, R. E. Continetti, and C. C. Hayden, J. Chem. Phys. 111, 1 (1999).

[13] O. Jagutzki, A. Cerezo, A. Czasch, R. Dorner, M. Hattas, M. Huang, V. Mergel, U. Spillmann, K. Ullmann-Pfleger, T. Weber, H. Schmidt-Bocking, and G. Smith, IEEE Trans. Nucl. Sci. 49, 2477 (2002).

[14] J. Ullrich, R. Moshammer, A. Dorn, R. Dörner, L. P. H. Schmidt, and H. Schmidt-Böcking, Rep. Prog. Phys. 66, 1463 (2003).

[15] See the PImMS website http://pimms.chem.ox.ac.uk.

[16] C. Vallance, M. Brouard, A. Lauer, C. S. Slater, E. Halford, B. Winter, S. J. King, J. W. L. Lee, D. E. Pooley, I. Sedgwick, R. Turchetta, A. Nomerotski, J. J. John, and L. Hill, Phys. Chem. Chem. Phys. 16, 383 (2014).

[17] H. Stapelfeldt and T. Seideman, Rev. Mod. Phys. 75, 543 (2003).

[18] A. T. J. B. Eppink and D. H. Parker, Rev. Sci. Instrum. 68, 3477 (1997).
[19] D. W. Chandler and P. L. Houston, J. Chem. Phys. 87, 1445 (1987)

[20] A. Nomerotski, M. Brouard, E. Campbell, A. Clark, J. Crooks, J. Fopma, J. J. John, A. J. Johnsen, C. S. Slater, R. Turchetta, C. Vallance, E. Wilman, and W. H. Yuen, J. Instrum. 5, C07007 (2010).

[21] J. J. John, M. Brouard, A. Clark, J. Crooks, E. Halford, L. Hill, J. W. L. Lee, A. Nomerotski, R. Pisarczyk, I. Sedgwick, C. S. Slater, R. Turchetta, C. Vallance, E. Wilman, B. Winter, and W. H. Yuen, J. Instrum. 7, C08001 (2012).

[22] I. Sedgwick, A. Clark, J. Crooks, R. Turchetta, L. Hill, J. J. John, A. Nomerotski, R. Pisarczyk, M. Brouard, S. H. Gardiner, E. Halford, J. Lee, M. L. Lipciuc, C. Slater, C. Vallance, E. S. Wilman, B. Winter, and W. H. Yuen, in IEEE 10th International Conference on New Circuits and Systems (NEWCAS) 2012, 17-20 June 2012, Montreal, QC (IEEE, Piscataway, NJ, 2012), pp. 497-500.

[23] A. T. Clark, J. P. Crooks, I. Sedgwick, R. Turchetta, J. W. L. Lee, J. J. John, E. S. Wilman, L. Hill, E. Halford, C. S. Slater, B. Winter, W. H. Yuen, S. Gardiner, M. L. Lipciuc, M. Brouard, A. Nomerotski, and C. Vallance, J. Phys. Chem. A 116, 10897 (2012).

[24] M. Brouard, E. Halford, A. Lauer, C. S. Slater, B. Winter, W. H. Yuen, J. J. John, L. Hill, A. Nomerotski, A. Clark, J. Crooks, I. Sedgwick, R. Turchetta, J. W. L. Lee, C. Vallance, and E. Wilman, Rev. Sci. Instrum. 83, 114101 (2012).

[25] L. J. Frasinski, K. Codling, and P. A. Hatherly, Science 246, 1029 (1989).

[26] W. A. Bryan, J. H. Sanderson, A. El-Zein, W. R. Newell, P. F. Taday, and A. J. Langley, J. Phys. B: At., Mol. Opt. Phys. 33, 745 (2000).

[27] D. A. Card, E. S. Wisniewski, D. E. Folmer, and A. W. Castleman, J. Chem. Phys. 116, 3554 (2002).

[28] A. E. Boguslavskiy, J. Mikosch, A. Gijsbertsen, M. Spanner, S. Patchkovskii, N. Gador, M. J. J. Vrakking, and A. Stolow, Science 335, 1336 (2012).

[29] J. Mikosch and S. Patchkovskii, J. Mod. Opt. 60, 1426 (2013).

[30] J. Mikosch and S. Patchkovskii, J. Mod. Opt. 60, 1439 (2013).

[31] W. G. Cochran, Biometrics 13, 261 (1957).

[32] M. P. Johansson and J. Olsen, J. Chem. Theory Comput. 4, 1460 (2008).

[33] G. Garcia, L. Nahon, and I. Powis, Rev. Sci. Instrum. 75, 4989 (2004).

[34] L. J. Frasinski, P. A. Hatherly, and K. Codling, Phys. Lett. A 156, 227 (1991). 